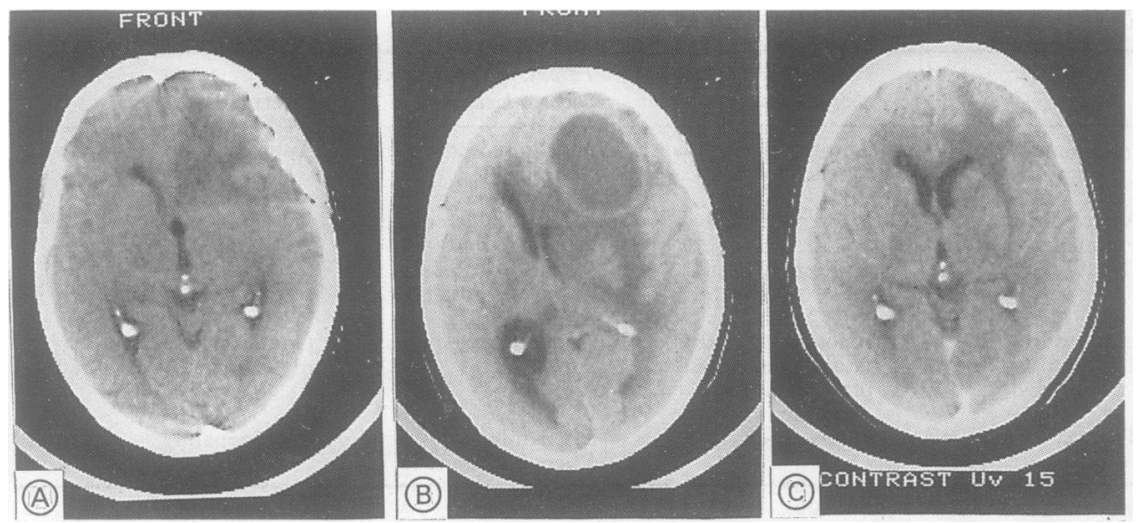

Figure A) CT without contrast showing low density area in the right frontal region; B) $C T$ without contrast showing cystic mass in the right frontal area which is enhanced; C) $C T$ scan at three months after aspiration showing low density area. There is no abscess and no shift.

primitive frontal reflexes slowly disappeared and she remained apyrexial. CT scans at weekly intervals showed no re-accumulation (figure $c$ ) and she regained her normal state of health.

The risk of infection after craniotomy is between $1-3 \%$ but after surgery from aneurysm, is less than $1 \%$. Cerebral abscesses are common secondary to otorhino-laryngo-logical infection. ${ }^{1}$ In this case craniotomy did not pass through an infected sinus. Among other causes are haematogenous spread in patients with cyanotic congenital heart disease and following oesophageal dilatation. ${ }^{23}$ In our case, the infection was most likely introduced at the time of operation. The mortality from intra-cerebral abscess ranges between $10-70 \%$ in untreated patients. The surgical management options include: tube drainage, masupialisation, Kahn's migration method, tapping, aspiration and excision. At presen only two methods are used, aspiration and excision. ${ }^{4}$ Excision of the abscess cavity carries a high risk of mortality and morbidity.

\section{MRI in hemiballism due to Sydenham's chorea}

Parainfectious and immunologically mediated chorea may be caused by a cytotoxic reaction in the basal ganglia. ${ }^{2}$ We report the MRI appearances in a patient with hemiballism due to Sydenham's chorea.

A 17 year old woman showed right sided hemiballism of subacute onset. She had a history of hyperthyroidism and repeated tonsillitis, but no family history of neurological diseases. Routine laboratory investigations showed normal blood cell counts and blood chemistry. Positive immunological findings were c-reactive protein (CRP) 16.5 (normal; $<0.3$ ), anti-streptlysin-O (ASO) 181 (<155), anti-streptokinase (ASK) $2560(<640)$ and decreased IgA of $32.9 \mathrm{mg}$ dl (135-340). There were no abnormal findings in anti-virus titres, hormonal levels, CSF and ECG. Although her involuntary movements were reduced by administration of sodium valporate and phenytoin, phenytoin caused an allergic agranulocytosis which required treatment with prednisolone, $40 \mathrm{mg}$ daily for 14 days. Prednisolone alleviated both the drug allergy and involuntary movements. After withdrawal of prednisolone
An intracerebral abscess after clipping of an aneurysm is rare. Our case demonstrates that they can be managed by minimal surgery and antibiotics without the need to remove the clip.

F M KAPASI Department of Neurosurgery,
Walton Hospital, Liverpool

Correspondence to: Dr Kapasi, Ysbyty Glan Clwyd, Bodelwyddan, Rhyl, Clwyd.

1 Arseni C, Ciurea AV. Cerebral abscesses secondary to otorhinolaryngological infections. $1988 ; 49(1): 22-36$.

2 Chakraborty R, Bidwai PS, KakVK, et al. Brain abscess in cyanotic congenital heart disease. Indian Heart f 1989;41(3): 190-3.

3 Pencek TL, Burchiel KJ. Delayed brain abscess related to a retained foreign body with culture of Clostridium bifermentans. Case report. $\mathcal{F}$ Neurosurg 1986;64(5):813-5.

4 Surgical treatment of brain abscess. Neurosurgery 1988;22(4);724-30.

5 Maurice Williams RS. Experience with “Open evacuation of pus" in the treatment of intracerebral abscess. Br $\mathcal{f}$ Neurosurg 1987;1(3): 343-51. haloperidol $5 \mathrm{mg}$ IM followed by $2.25 \mathrm{mg}$ daily by mouth for 20 days and the hemiballism resolved during this time. CT scan showed left caudate nucleus swelling only, and angiography was normal. T2 weighted MRI 31 days after onset of symptoms demonstrated hyperintensities in the left caudate nucleus, putamen, lateral pallidum, perirubral area and substantia nigra (Figure). Hyperintensity in the prerubral area suggested involvement of the subthalamic nucleus or its connecting pathways. "Fourteen months later, she had a convulsion with mental confusion. No ballistic movements were observed and a repeat MRI was normal. There were bursts of theta and delta wave activity in the EEG. Serological test showed positive antinuclear antibody (ANA: X320, speckled type) and negative anti-DNA antibody.

Despite the fact that systemic chorea is associated with systemic disease, unilateral involuntary movement are often seen. ${ }^{2}$ The lesions in our case seemed to spread during neural connections rather than in a vascular territory. The lesions were oedematous but was unlike lesions seen in vascular accidents or multiple sclerosis plaques. An allergic A study of 386 cases. Zentralbl-Neurochir

hemiballism reappeared and was treated with
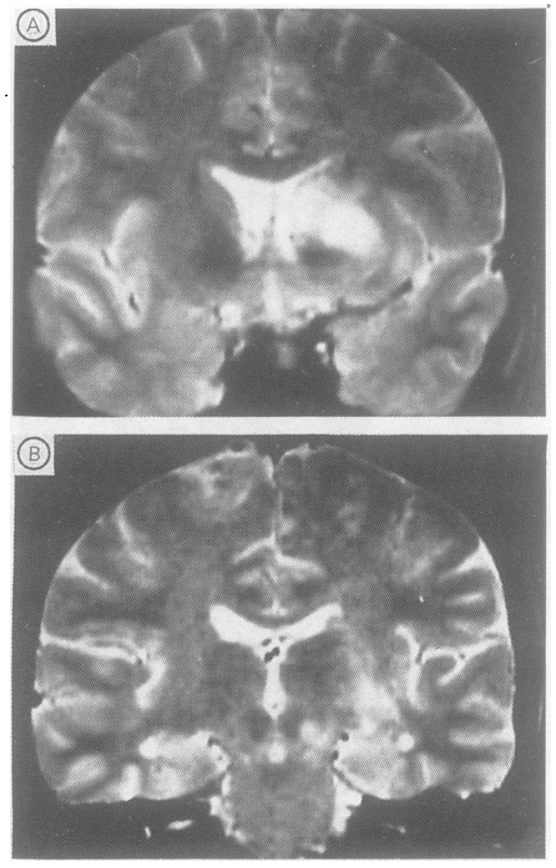

Figure T2 weighted MRI (TR 2000, TE 100). A: Elevated signal intensity area is observed in the caudate nucleus, putamen and lateral pallidum on the left. B: Elevation of signal intensity is also observed in the perirubral area and substantia nigra on the lefi.

reaction to phenytoin, amelioration of hemiballism by prednisolone and positive ANA, which appeared later, suggest an immunologically mediated disorder. The history of repeated tonsillitis and elevated ASO and CRP indicated the diagnosis of Sydenham's chorea. Sydenham's chorea is thought to be an immunologically mediated reaction in the basal ganglia and subthalamic nucleus. ${ }^{3}$ Increased signal intensity in T2 weighted MRI at an early stage suggests an exudative or inflammatory lesion, which is compatible with the hypothesis of an immunological reaction in the basal ganglia.

Besides lesions of the subthalamic nucleus, hemiballism can be caused by single or combined lesions of the striatum, pallidum, substantia nigra, thalamus and their connections in each other. ${ }^{45}$ Where the pallidum is affected, the lesion is usually in the outer segment. Disinhibition of the medial pallidum by the lesion of the inhibitory subthalamopallidal pathway and a subthalamic nucleus itself is thought to cause ballism.s MRI of our case showed a wide-spread lesion in the basal ganglia and related structures on the left. Of these, the perirubral area, where the subthalamopallidal pathway is located, and lateral pallidum were thought to be responsible for hemiballism.

Radiological findings of symptomatic chorea have been poorly documented. MRI of the present case offers the information about distribution and possible nature of the lesion.

$$
\begin{array}{r}
\text { MASAAKI KONAGAYA } \\
\text { Department of Neurology, } \\
\text { Suzuka National Hospital, Suzuka } \\
\text { YOKO KONAGAYA } \\
\text { Department of Neuroradiology, } \\
\text { Nagoya University School of Medicine, } \\
\text { Nagoya, fapan }
\end{array}
$$

Correspondence to: Dr Konagaya 
1 Nausieda PA. Sydenham's chorea, chorea gravidarum and contraceptive-induced chorea. Handbook of clinical neurology, vol 5(49) Extrapyramidal disorders. Amsterdam: Elsevier, trapyramidal

2 Padberg GW, Bruyn GW. Chorea - differential diagnosis. In: Vinken PJ, Bruyn GW, Klawans HL, eds. Handbook of clinical neurology, vol 5(49) Extrapyramidal disorders. Amsterdam: Elsevier, 1986:549-64.

3 Husby G, van de Rijn I, Zabriskie JB, Abdin $\mathrm{ZH}$, Williams RC. Antibodies reacting with cytoplasm of subthalamic and caudate nuclei neurons in chorea and acute rheumatic fever. f Exp Med 1976;144:1094-110.

4 Schwarz GA, Barrows LJ. Hemiballism without involvement of Luy's body. Arch Neurol 1960;2:420-34.

5 Buruma OJS, Lakke JPWF. Ballism. In: Vinken PJ, Bruyn GW, Klawans HL, eds. Handbook of clinical neurology, vol 5(49) Extrapyramidal of clinical neurology, vol 5(49) Extrapyramidal
disorders. Amsterdam: Elsevier, 1986: 369-80.

\section{Cervical spinal extradural haematoma causing a transient Brown-Sequard syndrome}

Extradural haematomas of the spinal canal are uncommon. They may be traumatic or occur spontaneously. Spontaneous recovery is exceptional. We report an extradural haematoma of the cervical spinal canal, precipitated by trauma, which resulted in a transient Brown-Sequard syndrome.

A previously fit 20 year old man developed an extradural haematoma in his cervical spinal canal after an episode of "disco dancing" and "head banging". The following day he complained of neck pain, and 36 hours after this, he presented to hospital complaining of weakness of his left arm and leg with sensory loss in his right leg. There was no previous history of trauma, neurological disease or bleeding disorder.

Physical examination on admission to hospital demonstrated a grade $2 / 5$ spastic weakness affecting the left side of the body below a level of C4. There was anaesthesia to pinprick below T8 on the right, and a right Horner's syndrome was noted. Bladder sensation and function were intact. Plain radiographs of the cervical spine were normal. A
CT scan of the cervical spine showed an extradural haematoma localised to the left side of the spinal canal between the levels of C4 and C6 (figure). Routine full blood count, film and clotting studies were normal.

The patient was treated with oral dexamethasone on admission and the neck immobilised in a cervical collar. The motor weakness improved over the subsequent 24 hours, followed by return of temperature sensation to the leg. Over the next three days the sensory level to pinprick sensation fell to the mid-lumbar dermatomes and then resolved completely. Steroid therapy was gradually withdrawn and the patient became ambulant without resurgence of his symptoms.

A Brown-Sequard pattern of dissociated sensory loss is extremely uncommon as a result of an extradural haematoma in the spinal canal. The first case was described in 1958 by Geuna and Pazni.' Since then a further four cases have been reported. ${ }^{2-5}$ These cases were all spontaneous haematomas occurring without preceding trauma. Two of these cases were associated with bleeding diatheses. ${ }^{45}$

Surgical intervention is advocated for most cases, especially when acute retention of urine has occurred. The cases of BrownSequard syndrome referred to were all associated with haematomas of the cervical canal, and all made good recoveries following decompressive laminectomy.

The case we have reported improved rapidly on steroids. By the time the patient had reached the Regional Neurological Centre in Newcastle, approximately 20 hours after first presenting to hospital, the neurological signs were receding. Conservative therapy was therefore continued and the patient made a complete recovery over the ensuing three days.

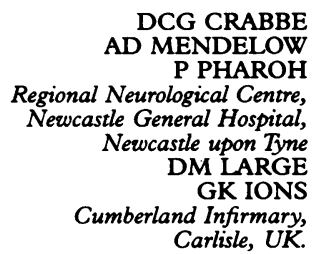

Correspondence to: Mr DCG Crabbe, Department of Pathology, The Royal Victoria Infirmary, Newcastle upon Tyne NE1 4LP

1 Geuna E, Pazni CA. Considerazioni se di un caso di compromissione midollasa da emotoma extradurale cronico. Rassegna Medica Sardinia 1958;60:645-9.

2 Segelov JN. Spinal epidural haematoma, a report of two cases. Pacific Medicine and Surgery 1967;75:169.

3 Russman BS, Kazi KH. Spinal epidural haematoma and the Brown-Sequard syndrome. Neurology (Minneapolis) 1971;21:1066-8.

4 Nattle H, Sieb JP, Rohner M, Mumenthaler M. Non-traumatic spinal epidural and subdural haematomata. Neurology 1987;37:1351-6.

5 Mustafa MH, Bernstein B. Spontaneous spinal epidural haematoma, Brown-Sequard syndrome and factor IX deficiency. Annals of Internal Medicine 1987;106:477-8.

Figure (A-left) Axial CT scan through C4 showing hyperdense right sided extradural haematoma in the spinal canal. (B-right) Axial CT scan through C5 (below) and sagittal reconstruction scan (above) demonstrating the length and posterior position of the extradural haematoma. 